\title{
An Algorithm for Estimating Liquid Flow Field From PTV Measurement Data of Bubble Motion*
}

\author{
Wen $\mathrm{CHENG}^{1}$, Yuichi MuraI ${ }^{2}$, Masa-aki Ishikawa ${ }^{3}$ and Fujio Yamamoto ${ }^{4}$
}

\begin{abstract}
In bubbly two-phase flow, gas phase and liquid phase have different flow fields, respectively. The relative velocity between two phases depends on the motion characteristic of bubbles. The mathematical expression for the motion of small bubble at low Re number is already established. Using the equation, liquid velocity along the trajectory of the bubble is inversely calculated by the motion equation. Whole field liquid flow structure is also estimated using spatial and/or temporal interpolation method. This paper proposes such an algorithm for estimating liquid phase flow field from measurement data of bubble motion. The frequency response analysis implies high advantage of the inverse analysis from bubble to liquid rather than solid particle to liquid. The applicability of the algorithm is examined with Taylor-Green vortex flow as an analytical test case, and with a bubbly plume as an experimental demonstration.
\end{abstract}

Keywords : Multiphase flow, Bubble, PIV, PTV, Inverse analysis, Computational fluid dynamics

\section{Introduction}

Particle image velocimetry (PIV) and particle tracking velocimetry (PTV) have rapidly advanced and spread in last decade ${ }^{1)}$. They have been applied to disperse multiphase flows in order to understand the physics of dispersion motion and the interaction between two phases. Through the theoretical research on bubble dynamics, the motion equations for a low Re number spherical bubble $(\operatorname{Re}<200)$ are almost established nowadays. Using the equation, the liquid flow can be estimated with the data of bubble motion in principle. Since PIV and PTV can measure time-dependent multi-dimensional bubble velocity vectors, it is expectable that the carrier phase flow is reconstructed with certain accuracy. This paper concerns such an algorithm based on recent knowledge of bubble dynamics and the technique of PIV including its post-processing.

The motion equation for a bubble ordinarily consists

* Paper received Feb., 6, 2003

1 University of Fukui (Bunkyo 3-9-1, Fukui 910-8507, Japan, E-mail: cheng@fv.mech.fukui-u.ac.jp) also holding the post of Xi'an Unversity of Technology, Xian 70048, P.R. China

2 Regular member, Graduate Sch. Eng., Hokkaido University

3 Nuclear Eng. Res. Labo., The University of Tokyo.

4 Regular member, Dept. Mech. Eng., University of Fukui of seven force components, i.e., inertia, added inertia, drag, lift, pressure gradient, gravity, and history forces. Among these components, lift is the one of unclear components. Sridhar-Katz ${ }^{2)}$ measured the lift force acting on a bubble using three-step PTV. Kitagawa et al. ${ }^{3)}$ measured a bubble-bubble interaction force in wall-sliding bubbles from the PTV data of bubble motions. Their research based on PIV and PTV provided useful information for improving the CFD model. On the other hand, the interpolation schemes for discrete data of velocity vectors have been proposed recently ${ }^{4)}$. Such the postprocessing schemes are also applicable to the reconstruction of carrier phase in bubbly flow.

The original point of our work lies on adequate combination of the mathematical model of bubble dynamics, application of PIV, and the post-processing. This kind of technique will be available for estimating the liquid flow in actual industrial systems, such as chemical reactor, heat exchanger, and naval environmental engineering, where the bubble motion is visible but too many tracer particles cannot be seeded to visualize the liquid flow.

\section{Basic Equations}

\section{1 Translation motion equation of single bubble}

Seven kinds of force vectors act on a bubble as it sus- 
pends in Newtonian liquid flow as follows.

$$
\mathbf{F}_{i}+\mathbf{F}_{g}+\mathbf{F}_{s}+\mathbf{F}_{a}+\mathbf{F}_{d}+\mathbf{F}_{l}+\mathbf{F}_{h}=0
$$

This equation consists of inertia force $\mathrm{F}_{i}$, gravity force $\mathrm{F}_{g}$, surface force $\mathrm{F}_{s}$, added inertia force $\mathrm{F}_{a}$, drag force $\mathrm{F}_{d}$, lift force $\mathrm{F}_{l}$, and history force $\mathrm{F}_{h}$. Mathematical formulation of each force component is nowadays well established for a low Re number spherical bubble in incompressible fluid as follows:

$$
\begin{aligned}
& \mathbf{F}_{i}=\rho_{g} V_{g} \frac{d \mathbf{u}_{g}}{d t} \\
& \mathbf{F}_{g}=\rho_{g} V_{g} \mathbf{g} \\
& \mathbf{F}_{s}=-\rho_{l} V_{g} \frac{d \mathbf{u}_{l}}{d t}-\rho_{l} V_{g} \mathbf{g} \\
& \mathbf{F}_{a}=\beta \rho_{l} V_{g}\left(\frac{d \mathbf{u}_{g}}{d t}-\frac{d \mathbf{u}_{l}}{d t}\right) \\
& \mathbf{F}_{d}=C_{D} \frac{1}{2} \rho_{l} \pi r_{g}{ }^{2}\left|\mathbf{u}_{g}-\mathbf{u}_{l}\right|\left(\mathbf{u}_{g}-\mathbf{u}_{l}\right) \\
& \mathbf{F}_{l}=C_{L} \rho_{l} V_{g}\left(\mathbf{u}_{g}-\mathbf{u}_{l}\right) \times\left(\nabla \times \mathbf{u}_{l}\right) \\
& \mathbf{F}_{h}=\mathbf{0}
\end{aligned}
$$

Where, $\rho, \mathbf{u}$, and $\mathbf{g}$ stand for density, velocity vector, and acceleration of gravity, respectively. Subscripts $g$ and $l$ stand for gas and liquid phases, respectively. $\mathrm{V}_{\mathrm{g}}$ stands for volume of a bubble. The surface force in Eq. (4) equals to the force due to pressure gradient and viscosity ${ }^{5)}$ in liquid replaced by Navier-Stokes equation. The added inertia force in Eq. (5) is expressed by Lagrangian acceleration components for two phases with added mass coefficient $\beta . C_{D}$ and $r_{\mathrm{g}}$ in Eq. (6) are drag coefficient and radius of bubble, respectively. The lift force in Eq. (7) is inertia lift force derived by Auton ${ }^{6)}$ with a lift coefficient $C_{L}$. History force is ignored in this study because it has a form of temporal integration that makes inverse analysis impossible. Nevertheless, it is actually negligible for a clean surface bubble at $\operatorname{Re}>50^{7)}$.

Substituting these formulae into Eq. (1) provides the following equation of motion.

$$
\begin{aligned}
\left(\rho_{g}+\beta \rho_{l}\right) & V_{g} \frac{d \mathbf{u}_{g}}{d t}=(1+\beta) \rho_{l} V_{g} \frac{d \mathbf{u}_{l}}{d t} \\
& +\left(\rho_{l}-\rho_{g}\right) V_{g} g \\
& -\left(C_{D} \frac{1}{2} \rho_{l} \pi r_{g}{ }^{2}\left|\mathbf{u}_{g}-\mathbf{u}_{l}\right|\left(\mathbf{u}_{g}-\mathbf{u}_{l}\right)\right. \\
& -C_{L} \rho_{l} V_{g}\left(\mathbf{u}_{g}-\mathbf{u}_{l}\right) \times\left(\nabla \times \mathbf{u}_{l}\right)
\end{aligned}
$$

Denoting the density ratio of gas to liquid by $\gamma$, Eq. (9) is simply expressed by:

$$
\begin{aligned}
(\gamma+\beta) \frac{d \mathbf{u}_{g}}{d t} & =(1+\beta) \frac{d \mathbf{u}_{l}}{d t}+(1-\gamma) \mathbf{g} \\
& -\frac{3 C_{D}}{8 r_{g}}\left|\mathbf{u}_{g}-\mathbf{u}_{l}\right|\left(\mathbf{u}_{g}-\mathbf{u}_{l}\right) \\
& -C_{L}\left(\mathbf{u}_{g}-\mathbf{u}_{l}\right) \times\left(\nabla \times \mathbf{u}_{l}\right)
\end{aligned}
$$

\section{2 Frequency response of translation motion}

Frequency response of a bubble for oscillatory flow of liquid is calculated by Eq. (10). Here, one-dimensional response is focused for simplicity while the density of gas is considered. Eq. (10) becomes linear in the case where $C_{D}$ is linearly proportional to $1 / R e$ (here, $R e=2$ $\left.\mathrm{r}_{\mathrm{g}}\left|\mathbf{u}_{\mathrm{g}}-\mathbf{u}_{l}\right| / v_{l}\right)$, such as Stokes or Levich's formula. Frequency response in this case is analytically solved as follows. When the liquid velocity is given by $\mathrm{u}_{l}=\mathrm{U}_{l} \sin$ $(2 \pi f t)$ with a frequency $f$, the bubble velocity behaves as $\mathrm{u}_{\mathrm{g}}=\alpha \mathrm{U}_{l} \sin (2 \pi f t+\phi)$. The ratio of velocity amplitude of gas to that of liquid is obtained as:

$$
\alpha=\left(1+2 \theta \cos \phi+\theta^{2}\right)^{-1}
$$

The phase lag of bubble is solved as:

$$
\tan \phi=\theta \sin \varepsilon(1+\theta \cos \varepsilon)^{-1}
$$

where, intermediate parameters $\theta$ and $\varepsilon$ are defined by:

$$
\left.\begin{array}{l}
\theta=(1-\gamma) f^{*}\left\{(\gamma+\beta)^{2} f^{*^{2}}+1\right\}^{-1} \\
\tan \varepsilon=\frac{1}{(\gamma+\beta) f^{*}}, f^{*}=\frac{16 r_{g}^{2} f}{3 A v_{l}}
\end{array}\right\}
$$

In above equations, $f^{*}$ is a dimensionless frequency. While density ratio is smaller than unity $(\gamma<1)$, it is quite important that the ratio of velocity amplitude $\alpha$ shifts from 1 to $(1+\beta) /(\gamma+\beta) \sim 3.0$ as frequency increases. Simultaneously, the phase lag is always negative, indicating the fact that bubble accelerates faster than liquid. The critical frequency at which $\alpha$ is central value between two states and the phase shift is the maximum, is given by:

$$
f_{c}^{*}=\{\sqrt{3}(\gamma+\beta)\}^{-1}
$$

For non-Stokes drag region, drag coefficient is nonlinear with respect to Reynolds number so that the dimensionless frequency cannot be defined. Assuming the bubble keeping completely spherical, the frequency response was obtained numerically for a $2 \mathrm{~mm}$ diameter air bubble in atmospheric pressure water using the following drag coefficients.

$$
\begin{aligned}
& C_{D}=\max \left\{\frac{16}{\operatorname{Re}}, \frac{48}{\operatorname{Re}}\left(1-\frac{2.21}{\sqrt{\operatorname{Re}}}\right)\right\} \\
& C_{D}=\frac{16}{\operatorname{Re}}\left(1+0.15 \operatorname{Re}^{0.687}\right)
\end{aligned}
$$




$$
C_{D}=\frac{24}{\operatorname{Re}}\left(1+0.15 \operatorname{Re}^{0.687}\right)
$$

Eq. (15) is combination of Stokes drag and Moore's $\mathrm{drag}^{8)}$. Eqs. (16) and (17) are for clean and contaminated bubble ${ }^{9)}$, respectively.

Figs. 1 and 2 show $\alpha$ and $\phi$ versus frequency f for four types of drag coefficients including Levich's drag ${ }^{8)}$ : 48/Re. Here the maximum velocity amplitude of liquid: $\mathrm{U}_{l}$ is $0.1 \mathrm{~m} / \mathrm{s}$, kinematic viscosity $v_{l}$ is $10^{-6} \mathrm{~m}^{2} / \mathrm{s}$, and surface tension $\sigma$ is $7.2 \times 10^{-2} \mathrm{~N} / \mathrm{m}$. Hence, Weber number defined by $W e=2 \mathrm{r}_{\mathrm{g}} \rho_{l} \mathrm{U}_{l}^{2} / \sigma$ is 0.27 . The data show that the critical frequency is lower for Levich's drag and Eq. (15) than Eqs. (16) and (17). Phase shift expands for Eqs. (15) and (16) bigger than linear drag cases. However, the order of the critical frequency is in the range of a few $\mathrm{Hz}$ in any case. Simultaneously, the phase shift is not negligible in this region. This result tells us that the amplification of bubble velocity often appears in engineering system and flow visualization experiment handling mm-order bubble flow. In Figs. 1 and 2 , gray bar indicates the frequency range of departure from spherical bubble in the actual situation, hence the data in this range do not express real one. In this

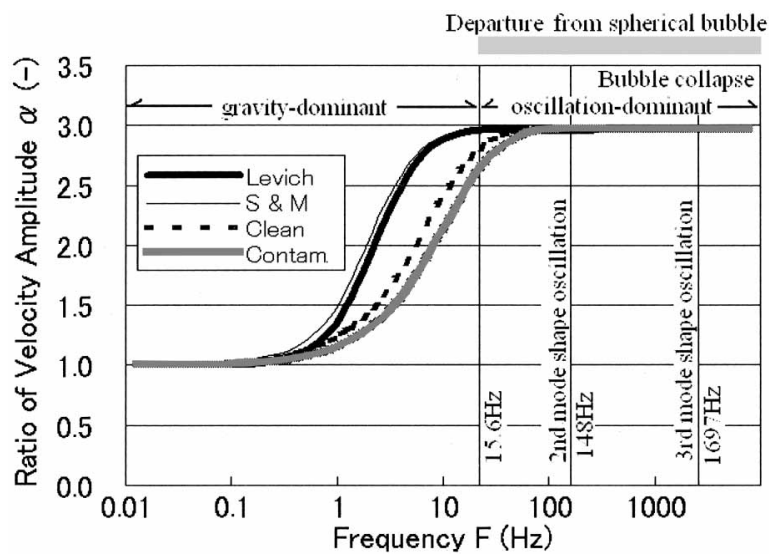

Fig. 1 Ratio of velocity amplitude versus frequency.

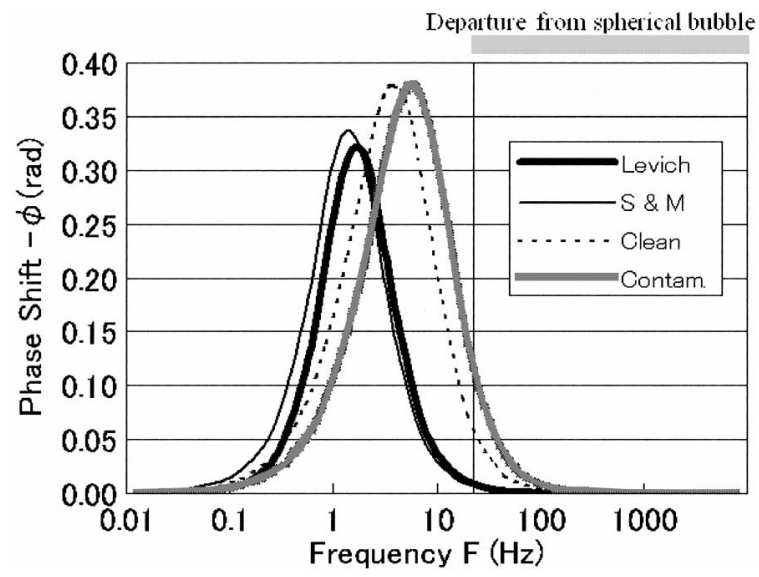

Fig. 2 Phase shift versus frequency. range the acceleration of liquid due to forced oscillation exceeds acceleration of gravity \{i.e.: $2 \pi \mathrm{fU}_{l}>\mathrm{g}$ \}. In addition, shape oscillation due to resonance grows at $148 \mathrm{~Hz}$ ( $2^{\text {nd }}$ mode: ellipsoidal) and $1697 \mathrm{~Hz}$ ( $3^{\text {rd }}$ mode: asymmetric deformation), at which the bubble may collapse as the oscillation continues. The resonance frequency $^{10)}$ is given by:

$$
f_{n}=\frac{1}{2 \pi}\left((n-1)(n+1)(n+2) \frac{\sigma}{\rho_{l} r_{g}^{3}}\right)^{\frac{1}{2}}
$$

where $n$ stands for the mode of shape oscillation. The detailed information on the deformation dependent oscillation of a bubble is obtained by the DNS of TakagiMatsumoto $^{9)}$. They found that the translational velocity amplitude was reduced more than the spherical bubble theory as the shape motion was induced. Anyhow, it is confirmed that the ratio of velocity amplitude for $1 \mathrm{~Hz}<$ $\mathrm{F}<10 \mathrm{~Hz}$, in which the bubble is spherical, is greater than unity in any cases of drag coefficient. In contrast, the particles with higher density than carrier fluid always behave at a little slower speed than the fluid in high frequency environment. From the sensitivity's point of view, thus the estimation of carrier phase velocity from bubble velocity is reasonable.

\section{3 Method of inverse analysis}

Eq. (10) can be transformed as follows.

$$
\begin{aligned}
& f_{p}\left(\mathbf{u}_{g}-\mathbf{u}_{l}\right)=(1-\gamma) \mathbf{g}+(1+\beta) \frac{d \mathbf{u}_{l}}{d t}-\mathbf{S} \\
& \mathbf{S}=(\gamma+\beta) \frac{d \mathbf{u}_{g}}{d t}+C_{L}\left(\mathbf{u}_{g}-\mathbf{u}_{l}\right) \times\left(\nabla \times \mathbf{u}_{l}\right)
\end{aligned}
$$

Where $f_{p}$ is a parameter with dimension of frequency given by:

$$
f_{p}=\frac{3 A v_{l}}{16 r_{g}{ }^{2}} g(\mathrm{Re})=f_{0} g(\mathrm{Re})
$$

here, $\mathrm{A}=16$ for clean bubbles, $\mathrm{A}=24$ for contaminant bubbles, and A = 48 in Levich's drag. $g(\mathrm{Re})$ is a function of Reynolds number defined by:

$$
g(\operatorname{Re})=C_{D}\left(\frac{A}{\operatorname{Re}}\right)^{-1}
$$

When liquid phase is quiescent and bubble rises with its terminal velocity, the liquid velocity at time $n$ can be calculated with:

$$
u_{l}^{n}=u_{g}{ }^{n}-\frac{(1-\gamma) g}{f_{p}^{n}}
$$

In transient case, the liquid velocity vector at time $n$ is given by:

$$
\mathbf{u}_{l}{ }^{n}=\frac{(1+\beta) \mathbf{u}_{l}{ }^{n-1}+\left\{a^{n-1} \mathbf{u}_{g}{ }^{n}-(1-\gamma) \mathbf{g}+\mathbf{S}^{n}\right\} \Delta t}{(1+\beta)+a^{n-1} \Delta t}
$$




$$
\begin{aligned}
\mathbf{S}^{n}= & (\gamma+\beta) \frac{\mathbf{u}_{g}{ }^{n}-\mathbf{u}_{g}{ }^{n-1}}{\Delta t} \\
& +C_{L}\left(\mathbf{u}_{g}{ }^{n-1}-\mathbf{u}_{l}{ }^{n-1}\right) \times\left(\nabla \times \mathbf{u}_{l}{ }^{n-1}\right)
\end{aligned}
$$

In the above equations, the bubble velocity at any moment is given by measurement. The liquid velocity in the past time $(n-1)$ is calculated at the former step. The time interval $\Delta \mathrm{t}$ corresponds with sampling time interval in measurement. When the lift force is ignored, i.e. $C_{L}=$ 0 in Eq. (25), the liquid velocity at each bubble position is easily obtained since the result will not be swayed by vorticity. When the lift force is taken into account, the liquid velocity must be interpolated at the former step to obtain the local vorticity surrounding each bubble.

For the interpolation of discrete velocity data of liquid phase, Laplace equation rearrangement (LER) method $^{4)}$ is applied for steady flow. Its extended version ${ }^{11)}$ for spatio-temporal interpolation method (ST-LER) can be applied for unsteady flow. The basic equations for these interpolation methods are as follows.

$$
\begin{aligned}
& \nabla^{2} \mathbf{u}_{l}=0 \\
& \kappa \frac{\partial^{2} \mathbf{u}_{l}}{\partial t^{2}}+\nabla^{2} \mathbf{u}_{l}=0 \\
& \mathbf{u}_{l}=\mathbf{u}_{l k} \delta+\mathbf{u}_{l}{ }^{*}(\delta-1)
\end{aligned}
$$

Eq. (26) is a Laplace equation for velocity vector $\mathbf{u}_{l}$ for steady flows. Eq. (27) is extended Laplace equation for unsteady flows. The parameter $\kappa$ in Eq. (27) is called spatio-temporal factor with the dimension of inverse velocity squared. The value of $\kappa$ is given by the squared ratio of the sampling time interval to the spatial sampling wavelength. Here, $\delta$ is delta function giving 1 at discrete data of $\mathbf{u}_{l k}$, and 0 at the position $\mathbf{u}_{l}{ }^{*}$ being interpolated.

Eventually, whole field flow structure of liquid phase in steady and transient states is estimated by the combination of Eqs. (24), (25), (27) and (28).

\section{Analytical Discussion}

In this section, the performance of the algorithm is discussed for a two-dimensional steady flow. As the test case, Taylor-Green vortex flow given by the following velocity components is chosen:

$$
\begin{aligned}
& u=W \sin \left(\frac{2 \pi k x}{L}\right) \cdot \cos \left(\frac{2 \pi k y}{L}\right) \\
& v=-W \sin \left(\frac{2 \pi k y}{L}\right) \cdot \cos \left(\frac{2 \pi k x}{L}\right)
\end{aligned}
$$

Here, $\mathrm{k}$ stands for wave number of vortex existing in measurement size $L . W$ is the amplitude of velocity.
Eqs. (29) and (30) satisfy equation of continuity. The bubbles are initially arranged randomly and their trajectories are calculated using periodic boundary condition. Of course the bubble diameter considered here must be much shorter than the wavelength of the vortex for validity of the translation motion of bubble. StokesMoore's drag coefficient by Eq. (15) is adopted but lift coefficient is set by $C_{L}=0$ for simplicity. It should be careful that the second term on right hand side in Eq. (19) is still required in steady flow since it is not local but Lagrange acceleration along bubble trajectory.

\section{1 Liquid velocity vector on bubble trajectories}

Bubble trajectory in Taylor-Green vortex is determined with two dimensionless parameters, $\mathrm{C}_{\mathrm{f}}=$ $f_{p} /(\mathrm{W} / \mathrm{L})$ and $\mathrm{C}_{\mathrm{g}}=\mathrm{g} /\left(\mathrm{W}^{2} / \mathrm{L}\right) . \mathrm{C}_{\mathrm{f}}$ stands for degree of friction between two phases, and $\mathrm{C}_{\mathrm{g}}$ stands for the ratio of gravity to the hydrodynamic force. Fig. 3(a) shows sample of bubbles' trajectories obtained at $C_{f}=9.0$ and $\mathrm{C}_{\mathrm{g}}=9.8$. These values are given assuming $W=1 \mathrm{~m} / \mathrm{s}, L$ $=1 \mathrm{~m}, g=9.8 \mathrm{~m} / \mathrm{s}^{2}$, and $f_{p}=9.0 \mathrm{~Hz}\left(v_{l}=10^{-6} \mathrm{~m}^{2} / \mathrm{s}, \mathrm{A}=\right.$ $48, r_{g}=1 \mathrm{~mm}$ for eq. (21)). The density ratio $\gamma$ is 0.01 . Gray segments stand for the liquid velocity vectors. Bubbles rise with meandering motion affected by the rotational motion of liquid. Fig. 3(b) shows the distribution of liquid velocity vectors estimated along the bubble trajectory. The orientation of the liquid velocity corresponds qualitatively with the original distribution shown in Fig. 3(a).

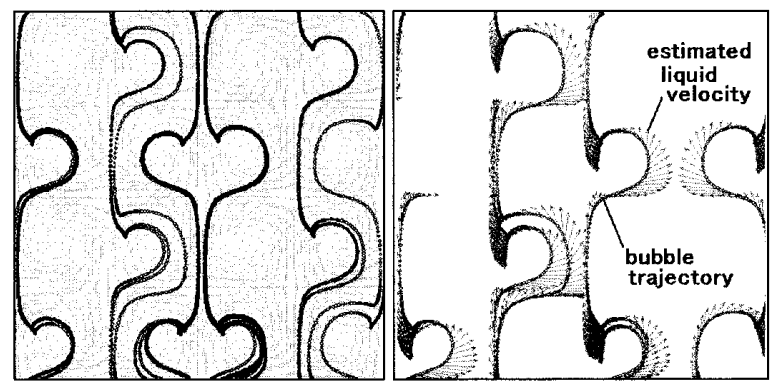

(a) Bubble trajectories

(b) Estimated liquid velocity

Fig. 3 Estimation of Taylor-Green vortex flow at $\mathrm{k}=2$.

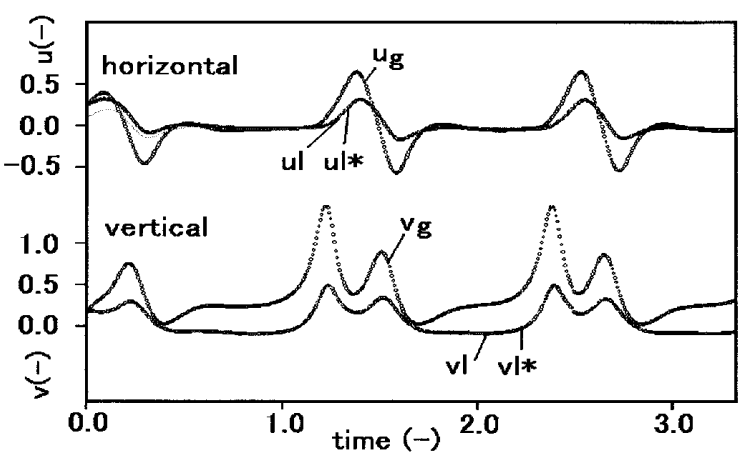

Fig. 4 Fluctuation of bubble and liquid velocity. 
Fig. 4 shows velocity fluctuation as a bubble rises in the same condition. Bubble velocity $\left(\mathrm{u}_{\mathrm{g}}, \mathrm{v}_{\mathrm{g}}\right)$ has larger amplitude in both directions than that of liquid $\left(\mathrm{u}_{l}, \mathrm{v}_{l}\right)$. The peak point is seen earlier for bubbles than that for liquid. These features correspond with the result of frequency response analysis mentioned in the former section. As a result, the estimated liquid velocity $\left(\mathrm{u}_{l}{ }^{*}\right.$, $\mathrm{v}_{l}{ }^{*}$ ) with the present algorithm completely agrees with the true value.

The liquid velocity vector just on the bubble path is successfully calculated, if the bubble position is tracked at a sufficiently short time interval. We have confirmed that no error was grown in any conditions of the parameters $k, \mathrm{C}_{\mathrm{f}}$ and $\mathrm{C}_{\mathrm{g}}$ if the bubble displacement between two time steps was sufficiently short compared to the minimum wavelength of the bubble trajectory. The factor to cause the error is only the numerical error of differentiation employed to Eqs. (19) and (20). Eq. (24) is a discretized form using implicit first order differentiation that ensures rather numerical stability at arbitrary time interval than temporal precision. It is similar to general knowledge of finite difference method, i.e., higher order differentiation is recommended to achieve high temporal resolution. The examination with a variety of differentiation schemes cannot be described for lack of space. Another problem arises from biased distribution of bubbles as resultant interaction between two phases. Even in this case the liquid velocity vector at the bubble existing position itself is calculated without any numerical instability.

\section{2 Estimation of whole liquid velocity vector field}

Liquid velocity vector field is interpolated from the discrete data on individual bubble using LER, i.e., Eq. (26), the whole flow field of the liquid phase can be reconstructed. In this process the discrepancy between the true flow and the reconstructed flow takes place dependent on the bubble distribution while the velocity vector of liquid at each bubble is accurate. Here, the performance of whole flow field estimation is evaluated with following cross correlation function:

$$
C=\frac{\sum\left(\phi_{t h} \cdot \phi_{R}\right)}{\sqrt{\sum \phi_{t h}{ }^{2} \cdot \sum \phi_{R}{ }^{2}}}
$$

Where $\phi$ is a target variable, suffix th stands for theoretical value (or original value), and suffix $R$ stands for reconstructed (or estimated) value. As the value of $C$ reaches 1.0 , it indicates that the estimated variable field becomes completely similar to the original field. In this examination, the cross correlation is calculated by the average of two values of $C$ for individual velocity compo- nents, $u$ and $v$.

Figs. 5 to 7 show the cross correlation coefficients with respect to three parameters. In all the cases, $f_{p} /(\mathrm{W} / \mathrm{L})$ and $\mathrm{g} /\left(\mathrm{W}^{2} / \mathrm{L}\right)$ are fixed at 9.0 and 9.8 , respectively since these parameters are environmental property rather than bubble's condition. The lift force is considered because the velocity gradient of liquid phase can be calculated with interpolated velocity field.

Fig. 5 confirms that the more the number of bubbles increases, the better the whole flow field is reconstructed. This feature itself comes from the basic performance of $\mathrm{LER}^{4)}$.

Fig. 6 tells us that the dispersion with an equal density to the fluid provides the better reconstruction also in the inverse analysis. The main reason for this fact is not the limitation of the inverse analysis methodology but it is the biased distribution of non-equal density dispersions in the flow field, i.e., bubbles tend to accelerate to vortices while heavy particles tend to move straightly beyond the small structure or stay in high strain rate regions. Sugiyama et $\mathrm{al}^{13)}$ discussed the feature of bubble distribution in Taylor-Green vortex flow. They found that the bubble path was fixed along selected curves at low Stokes number while that took arbitrary position at high Stokes number. This feature

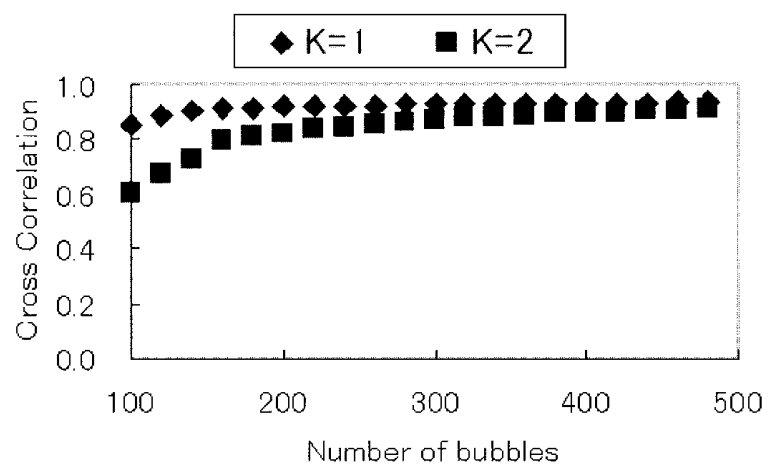

Fig. 5 Cross correlation vs. number of bubbles $\left(f_{p} /(\mathrm{W} / \mathrm{L})=9.0\right.$, $\gamma=0.01)$.

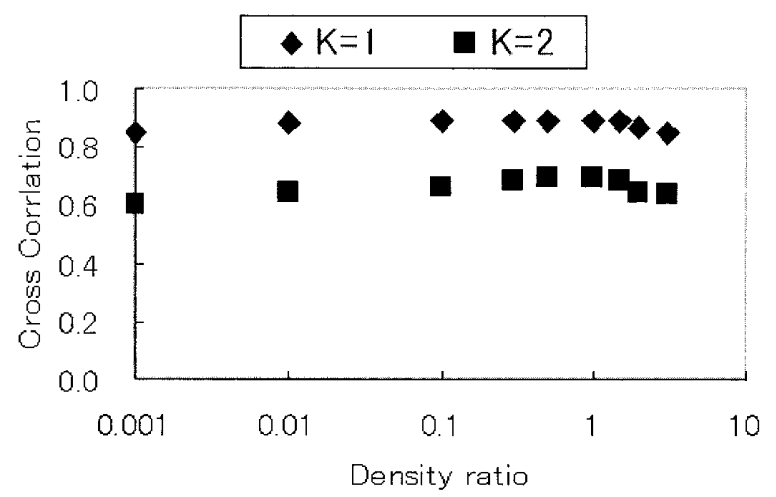

Fig. 6 Cross correlation vs. density ratio of dispersion $\left(f_{p} /(\mathrm{W} / \mathrm{L})\right.$ $=9.0, \mathrm{n}=0.01$ ). 


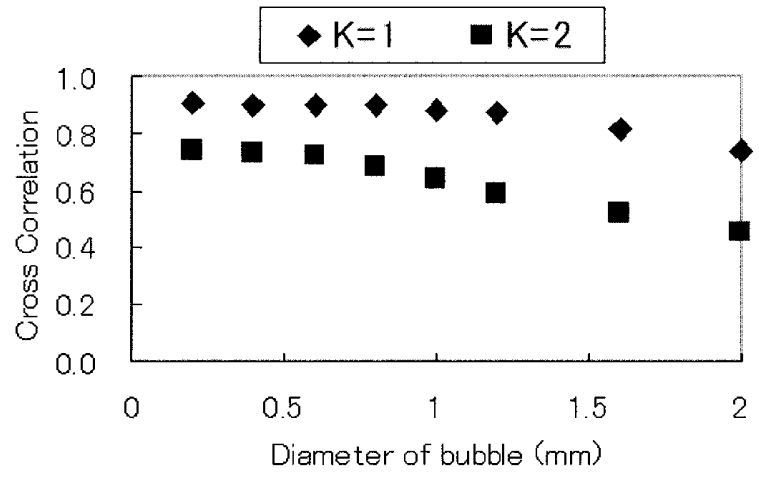

Fig. 7 Cross correlation vs. diameter of bubble $(\mathrm{n}=100, \gamma=$ $\left.0.01, f_{p} /(\mathrm{W} / \mathrm{L})=9.0\right)$.

strongly reduces the reconstruction performance for low St number cases, however, in the actual application, the bubble path scatters widely because of the size deviation and the unsteadiness of the flow.

It is confirmed with Fig. 7 that the flow field is better reconstructed for smaller bubbles dispersed. However the cross correlation keeps more than 0.8 in the case of a relatively large bubble up to $2 \mathrm{~mm}$ when k equals to 1 . The data mean the advantage of the inverse analysis from the bubble's measurement. Bubbles of more than $2 \mathrm{~mm}$ are not examined because those will cause intrinsic motion unrelated to local liquid flow such as zigzag or spiral behavior to make the inverse analysis impossible.

\section{Application to a Bubbly Two-Phase Flow}

\section{1 Application to a bubble plume}

The proposed method is applied for the actual measurement of a bubble plume as the demonstration. The bubble plume is generated in the rectangular tank of $0.30 \mathrm{~m}$ in width, and $0.80 \mathrm{~m}$ in height. Clean water and $\mathrm{N}_{2}$ gas are used as the media.

A sample of the experimental image is shown in Fig. 8(a). In this case, the bubble plume rises with a snaky motion initially and diffuses at the upper part. Such the asymmetric mixing behavior of the bubble plume is well known as the effect of large-scale interaction between two phases ${ }^{14)}$. The bubble velocity vectors are calculated with PIV as shown in Fig. 8(b), based on recursive cross correlation algorithm (pp. 95-97 in the handbook ${ }^{1)}$ ). The mean bubble diameter is $2.0 \mathrm{~mm}$, and the terminal velocity is $0.20 \mathrm{~m} / \mathrm{s}$. The local void fraction just above the bubble injector is 0.08 as averaged value for 0 $<\mathrm{y}<0.1 \mathrm{~m}$. The region at $\mathrm{y}>0.1 \mathrm{~m}$ has a void fraction lower than 0.05 according to image measurement result ${ }^{14)}$. The influence of bubble-bubble interaction that is ignored in this study arises only near the bubble injec-

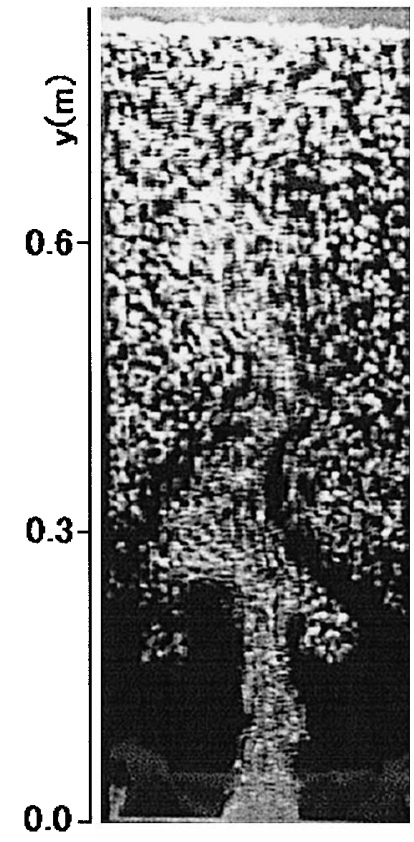

(a) Bubble image

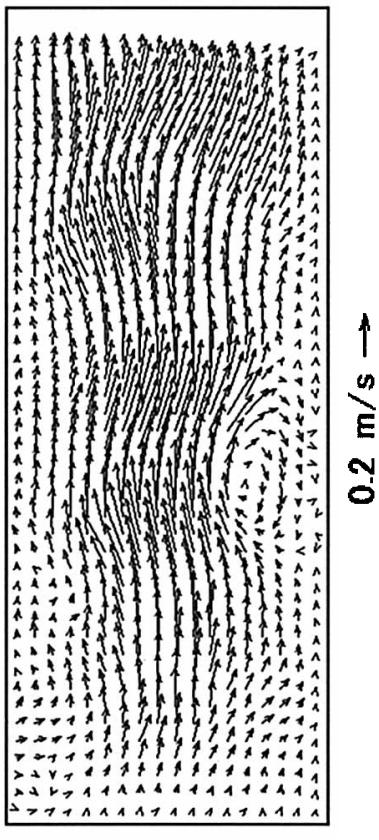

(b) Bubble velocity
Fig. 8 PIV measurement of bubble motion.

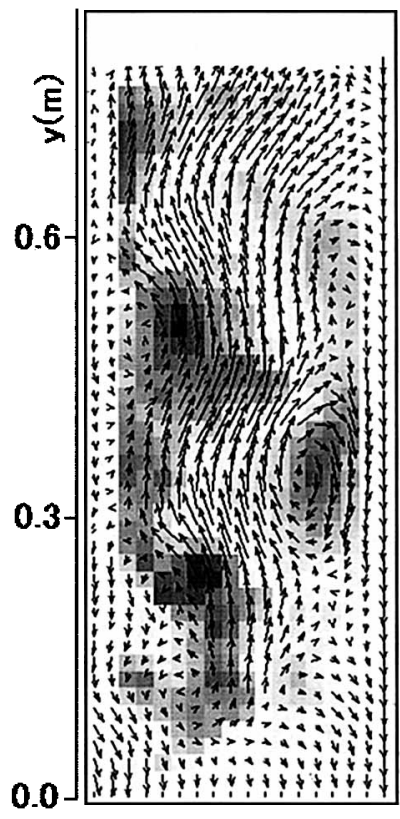

(a) Simple subtraction

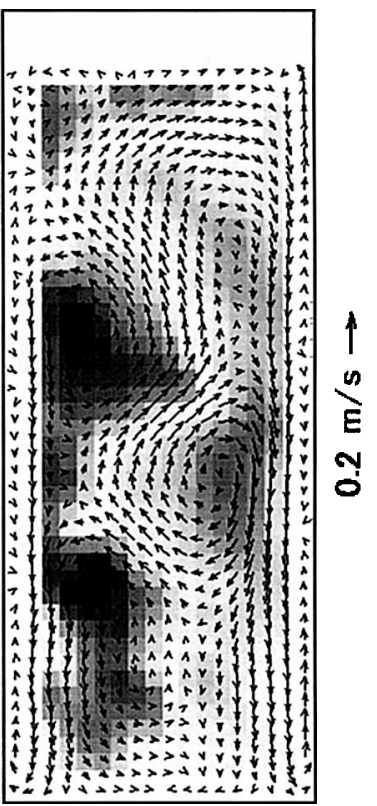

(b) Present method
Fig. 9 Estimated liquid flow field.

tor while it is negligible for $\mathrm{y}>0.1 \mathrm{~m}$.

Fig. 9 shows the estimated result of the liquid flow field expressed by the velocity vectors and the enstrophy with gray level. If the liquid velocity is simply estimated by subtracting the terminal rising velocity of the bubble, the liquid flow is calculated as shown in Fig. 9 (a). In this case, the local circulation of liquid is overestimated. When the inverse analysis is applied, the liquid flow is more smoothly obtained as shown in Fig. 9(b). 
In addition, the continuity of the convection is improved in the bottom and the top regions of the tank. The vorticity obtained by the present method validly indicates the re-circulation structure of the liquid ${ }^{14)}$. Estimating from the flow structure, the typical frequency of liquid velocity fluctuation acting on rising bubbles is around $1.0 \mathrm{~Hz}$ at which the velocity amplitude ratio is $1.2-1.5$ and the phase shift is $0.10-0.25$ rad as shown in Figs. 1 and 2.

\section{2 Applicable range of the present method}

The present method is applicable when the following five conditions are satisfied simultaneously:

1) Bubble is approximately spherical so that $\mathrm{We}$ number is less than 0.50 .

2) No self-excited behavior of bubble such as zigzag and spiral motion emerges, i.e. $\operatorname{Re}<200$. Also, Re number should be larger than 50 for ignoring the history force.

3) Local void fraction is less than $5 \%$ so that the influence of bubble-bubble interaction is negligible. The bubble-bubble interaction affects the flow field with a less void fraction in some cases but $5 \%$ corresponds with allowable relative error in the final result.

4) Typical spatial scale of liquid flow is much larger than bubble size. For instance turbulent eddy smaller than bubble cannot be reconstructed because of limitation to the expression of bubble motion by the translational motion of equation. According to the experience of CFD with Eulerian-Lagrangian model ${ }^{5)}$, the typical bubble size must be smaller than $1 / 10$ of vortex size.

5) Temporal resolution of measurement is enough high so that bubbles do not go across the minimum scale of the liquid flow in the sampling period.

6) Number of bubbles in a vortex is larger than 40 for the LER to interpolate the flow field with $80 \%$ of cross correlation. If its improved version ${ }^{4)}$ named BER (biquadratic ellipsoidal rearrangement) is employed, the lower limit decreases to around 10.

The estimated applicable range of the present method is summarized in Table $\mathbf{1}$ considering above-mentioned conditions. These conditions come not only from the validity of Eqs. (1)-(8), Eqs. (26)-(28) but also from empirical knowledge of PTV and FDM (finite difference method). Therefore these values are just rough estimates for judgment of utility. Even though these conditions are completely kept in the experimental application, the solution of the present method is never the exact answer but it is best-estimated structure of the carrier flow as mentioned in the $3^{\text {rd }}$ section.
Table 1 Applicable range of the present method

\begin{tabular}{|l|c|l|}
\hline & \multicolumn{1}{|c|}{ Min. } & Max. \\
\hline Bubble Re number & 50 & 200 \\
\hline Bubble We number & 0 & 0.50 \\
\hline Local void fraction & 0 & 0.05 \\
\hline Bubble diameter/Vortex size & 0 & 0.10 \\
\hline Number of bubbles/Vortex & $\begin{array}{l}40 \text { for LER } \\
10 \text { for BER }\end{array}$ & free \\
\hline
\end{tabular}

\section{Conclusion}

We proposed an algorithm for estimating the liquid phase flow from the measurement data of bubble motion. Through this work, the following remarks have been concluded.

1) Frequency response analysis for a spherical bubble in a forced oscillation revealed the advantage of the inverse analysis from the bubble to liquid from the sensitivity's point of view in the measurement.

2) In the analytical examination with Taylor-Green vortex flow, the velocity waveform of liquid along the bubble trajectory was perfectly extracted if the lift force of bubble was ignored.

3) Taking the lift force into account, the whole flow field of the vortex flow was successfully reconstructed by combining the inverse analysis and the interpolation schemes for particle tracking velocimetry.

4) As a demonstration, the liquid phase flow field was calculated from the PIV measurement result for the bubbles. The present method could estimate the flow better than a method simply subtracting the terminal velocity.

\section{References}

1) Visualization Soc. Japan edit: Handbook of Particle Image Velocimetry, Morikita Shuppan (2002) pp. 1-328 (in Japanese).

2) Sridhar, G., Katz, J.: Drag and Lift Forces on Microscopic Bubbles Entrained by a Vortex, Phys. Fluids, A, Vol. 7 (1995) pp. 389-398.

3) Kitagawa, A., Ashihara, M., Murai, Y., Yamamoto, F.: BubbleBubble Interaction Observed in a Swarm of Wall-Sliding Bubbles, Trans. JSME, B, Vol. 67, No. 657 (2002) pp. 11111118.

4) Ido, T., Murai, Y., Yamamoto, F.: Post-processing Algorithm for Particle Tracking Velocimetry Based on Ellipsoidal Equations, Exp. Fluids, Vol. 32 (2002) pp. 326-336.

5) Murai, Y., Matsumoto, Y.: Numerical Study of the ThreeDimensional Structure of a Bubble Plume, J. Fluids Eng., ASME, Vol. 122 (2000) pp. 754-760.

6) Auton, T.R.: The Lift Force on a Spherical Body in a Rotational Flow, J. Fluid Mech., Vol. 183 (1987) pp. 199-220. 
7) Takagi, S., Matsumoto, Y.: Force Acting on a Spherical Bubble Rising in Quiescent Fluids, Japanese J. Multiphase Flow, 10, (1996) pp. 264-273 (in Japanese).

8) Moore, D.W.: The Rise of a Gas Bubble in a Viscous Liquid, J. Fluid Mech., Vol. 6 (1959) pp. 113-133.

9) Tomiyama, A., Kataoka, I., Zun, I., Sakaguchi, T.: Drag Coefficients of Single Bubbles under Normal and Micro Gravity Conditions, JSME Int. J., B., Vol. 41 (1998) pp. 472-479.

10) Leal, L.G., Laminar Flow and Convective Transport Processes, Butterworth, Boston (1992) pp. 331-331.

11) Takagi, S., Matsumoto, Y.: The Behavior of a Deformable
Bubble in an Accelerating Fluid, Trans. JSME, B, 64-624 (1998) pp. 2483-2490 (in Japanese).

12) Murai, Y., Ido, T., Yamamoto, F.: Post-Processing Method Using Ellipsoidal Equations for PTV Measurement Results, JSME Int. J., B, Vol. 45 (2002) pp. 142-149.

13) Sugiyama, K., Takagi, S., Matsumoto, Y.: Translational Motion of Bubbles and Particles in Cellular Flow, Trans. JSME, B, 69-680 (2003) pp. 786-793 (in Japanese).

14) Murai, Y., Matsumoto, Y., Yamamoto, F.: Qualitative and Quantitative Flow Visualization of Bubble Motions in a Plane Bubble Plume, J. Visualization, Vol. 3 (2000) pp. 27-35. 